\title{
Characterisation of major histocompatibility complex class I genes at the fetal-maternal interface of marsupials
}

Ina Buentjen ${ }^{1}$, Barbara Drews ${ }^{1}$, Stephen R Frankenberg ${ }^{2}$, Thomas B Hildebrandt ${ }^{1}$, Marilyn B Renfree $^{2}$ Brandon R Menzies ${ }^{2 \S}$

${ }^{1}$ Leibniz Institute for Zoo and Wildlife Research, Department of Reproduction Management, Alfred-Kowalke Strasse 17, Berlin, Germany.

${ }^{2}$ School of BioSciences, The University of Melbourne, 3010, Victoria, Australia.

${ }^{\S}$ Corresponding author $(\mathrm{Ph}:+6138344$ 7040)

Email addresses:

IB: ina.buentjen@gmail.com

BD: drews@izw-berlin.de

SRF: $\underline{\text { srfr@unimelb.edu.au }}$

TBH: hildebrandt@izw-berlin.de

MBR: m.renfree@unimelb.edu.au

BRM: menziesb@unimelb.edu.au 


\begin{abstract}
Major Histocompatibility Complex class I molecules (MHC-I) are expressed at the cell surface and are responsible for the presentation of self and non-self antigen repertoires to the immune system. Eutherian mammals express both classical and non-classical MHC-I molecules in the placenta, the latter of which are thought to modulate the maternal immune response during pregnancy. Marsupials last shared a common ancestor with eutherian mammals such as humans and mice over 160 million years ago. Since, like eutherians, they have an intra-uterine development dependent on a placenta, albeit a short-lived and less invasive one, they provide an opportunity to investigate the evolution of MHC-I expression at the fetal-maternal interface. We have characterised MHC-I mRNA expression in reproductive tissues of the tammar wallaby (Macropus eugenii) from the time of placental attachment to day 25 of the 26.5 day pregnancy. Putative classical MHC-I genes were expressed in the chorio-vitelline placenta, fetus and gravid endometrium throughout the whole of this period. The MHC-I classical sequences were phylogenetically most similar to the Maeu-UC (50/100 clones) and Maeu-UA genes (7/100 clones). Expression of three non-classical MHC-I genes (Maeu-UD, Маеи-UK and Маеи-UM) were also present in placental samples. The results suggest that expression of classical and non-classical MHC-I genes in extant marsupial and eutherian mammals may have been necessary for the evolution of the ancestral therian placenta and survival of the mammalian fetus at the maternal-fetal interface.
\end{abstract}

Key words: immune, mammal, ancestor, reproduction, placenta, viviparity 


\section{Background}

The vertebrate immune system has evolved an intricate system to identify and eliminate foreign cells and material, but preserve self-integrity (Zinkernagel and Doherty 1974). Major Histocompatibility Complex class I molecules (MHC-I) represent a diverse gene family that is vital for the immune system to differentiate between self and non-self and to eradicate infected cells (Zinkernagel and Doherty 1974; Hulpke and Tampé 2013). MHC-I molecules can be further divided into classical and non-classical types. Classical MHC-I are expressed on the surface of all nucleated cells (Bjorkman and Parham 1990). They bind and present intra-cellular peptides for cross-presentation with cytotoxic T-cells. The peptide-binding region (PBR) of MHC-I molecules allows for the binding of a large repertoire of peptides (Hedrick and Thomson 1988; Hughes and Nei 1988). If foreign, viral antigen is presented, the cells are targeted for destruction. The PBR of classical MHC-I molecules are highly polymorphic. In contrast, non-classical MHC-I molecules have low levels of PBR polymorphisms and are only present on a restricted subset of tissues, such as the placenta of eutherian mammals (Pyo et al. 2006). In addition to antigen presentation, nonclassical MHC-I play a role in immuno-modulation by inhibiting natural killer cells (Andrews et al. 2012).

Viviparity, or live birth, presents the vertebrate immune system with a unique challenge in that genetically dissimilar tissues (i.e. those of the mother and of the embryo) are in close apposition, and for lengthy periods of time in some species. An extended phase of internal development requires the transfer of nutrients, which is provided by the placenta. Mammalian placental morphology and the degree of invasiveness are highly diverse (Amoroso 1959). However, all of the anatomical variations seen in mammals today are believed to have evolved from that of a common ancestor of marsupial and eutherian mammals over 160 million years ago (Luo et al. 2011).

One of the most recognisable differences between the two subclasses of therian mammals (eutherians and marsupials) is their relative investment in pregnancy. Relative to eutherian mammals, marsupials have short pregnancies during which the conceptus/es are enveloped by a maternally secreted shell coat for $60-80 \%$ of total pregnancy length and maternal investment in pregnancy is much less (Tyndale-Biscoe and Renfree 1987; Selwood 2000; Renfree et al. 2009). Depending on the species, direct fetal apposition, and or invasion of the maternal endometrium, does not occur until after the shell coat degrades in the second half of pregnancy (Tyndale-Biscoe and Renfree 1987). In contrast, eutherian embryos generally make contact with, and in many cases, invade the endometrium at an early stage of development. Furthermore, the fetus is retained in utero 
for a relatively longer period of development so that in some precocial species the young is already semi-independent at birth (eg guinea pigs).

Since maternally and paternally derived MHC alleles are expressed in a co-dominant pattern, the support of the partially foreign fetus during pregnancy remains an enigma (Medawar 1953; Bainbridge 2000). Absence of classical MHC-I expression in embryonic tissues that are in direct contact with the mother may be one adaptation to avoid maternal immune recognition (Bainbridge 2000). Human extra-villous trophoblast, which is surrounded by maternal blood, does not express the highly polymorphic classical MHC-I $H L A-A$ and $-B$, but does express $H L A-C$, which has lower levels of PBR polymorphism (Redman et al. 1984; Parham et al. 2012). HLA-C is a killer-cell immunoglobulin-like receptor (KIR) ligand and is thought to modulate natural killer cell activity in human trophoblast (Chazara et al. 2011). In contrast, classical MHC-Is are expressed by all nontrophoblastic cells and the maternal decidua (Blaschitz et al. 2001). Human villous trophoblast and preimplantation embryos also express non-classical, immune-modulatory MHC-I, HLA-E and HLA$G$ (Cao et al. 1999).

Bovine classical MHC-Is are not expressed by trophoblast cells before day 120 of gestation (gestation length: 240 days) (Davies et al. 1990). Equine trophoblast also appears to have reduced classical MHC-I protein localisation relative to the apposing endometrium (Donaldson et al. 1990). Mice express the classical MHC-I $H 2-K$ and $H 2-D$ in trophoblast, and paternal:maternal mismatch in $H 2-K$ and $H 2-D$ MHC-I alleles results in greater maternal uterine vascularisation and placental and fetal growth, suggesting, at least in this species, that the level of dissimilarity between maternal and fetal MHC-I actually promotes reproductive success (Madeja et al. 2011). While mouse nonclassical MHC-I molecules have been detected in crude placental extracts, more recent studies have demonstrated an absence of non-classical MHC-I cell-surface expression in mouse trophoblast (Warner et al. 1987; Madeja et al. 2011).

Classical MHC-I genes are subject to rapid evolution through gene duplication and divergence so orthologous relationships are not easily resolved between distant mammalian lineages (Rogers 1985; Nei et al. 1997). Thus, MHC-I characterisation in a new species can require extensive genome sequencing and analysis to infer function so there are relatively few species in which both the molecular and functional characteristics of these molecules are defined. The MHC loci of humans and mice have been very well described, while that of zebrafish (Sambrook et al. 1995), frog (Nonaka et al. 1997), chicken (Shiina et al. 2004), pig (Gaycken et al. 1994), rat (Günther and Walter 2001), horse (Gustafson et al. 2003; Tallmadge et al. 2005), rhesus macaque (Otting et al. 
2005), cow (Birch et al. 2006), dog, cat (Yuhki et al. 2006), and three marsupials including opossum (Belov et al. 2006), tammar wallaby (Siddle et al. 2009) and Tasmanian devil (Siddle et al. 2010).

Relative to opossum and Tasmanian devil, tammar wallaby MHC-I genes have undergone duplication, translocation and diversification within the genome such that they are scattered across the seven autosomes that make up the tammar karyotype. Interestingly, some MHC-I genes, including the putative classical genes, have moved to a genomic location away from the main MHC gene cluster on chromosome two (MHC-unlinked) (Siddle et al. 2009). This is different from that described in most vertebrate species in which all MHC-I genes remain within the MHC gene cluster. Three MHC-I genes (Maeu-UA: Ch7, Maeu-UB: Ch6, and Maeu-UC: Ch1) have been identified as putative classical genes on the basis of strong, ubiquitous tissue expression, PBR polymorphism, and conserved promoter elements relative to the classical MHC-I of eutherian mammals. By contrast, putative non-classical genes are located both within (Maeu-UK, Maeu-UM, Maeu-UE Maеu-UL, Маеи-UP, and Maeu-UO: Ch2) and away (Maeu-UD and Maeu-UN: Ch5, Maeu-UF and Maeu-UH: Ch4, Maeu-UI: Ch7, Maeu-UJ: Ch1) from the MHC gene cluster, show tissue-specific expression, low PBR sequence polymorphism and non-conserved promoter sequences (Siddle et al. 2009). Maeu-UI, -UJ, -UL, and -UP genes show no evidence of expression. To date, no study has characterised MHC-I expression in reproductive tissues including the placenta of any marsupial.

The tammar wallaby possesses a non-invasive, chorio-vitelline or yolk sac placenta that directly apposes the maternal endometrium after shell-coat rupture at approximately day 18 of the 26-day active pregnancy (Tyndale-Biscoe and Renfree 1987). The fetal part of the placenta comprises two parts, the bilaminar (BOM) and trilaminar (TOM) omphalopleure. While the TOM is composed of trophoblast, mesenchyme (including blood vessels) and endoderm, the BOM lacks mesenchymal tissue (Freyer et al. 2002). Tammars are seasonal breeders in which a single blastocyst reactivates from diapause during late December in one of two anatomically separate uteri. While the endometrium of both uteri is primed for pregnancy by progesterone from the corpus luteum, the endometrium of the gravid uterus proliferates to a greater extent due to the presence of the conceptus (Renfree 1972).

This study therefore characterises MHC-I expression and sequence diversity in the bilaminar and trilaminar placenta and the gravid and non-gravid endometrium of the tammar wallaby from somite stages at d18 to nearly full term fetuses at day 25 of the 26-day pregnancy. 


\section{Methods}

\section{Animals}

Female tammar wallabies were shot on agricultural land on Kangaroo Island, South Australia from mid-late January under approved permit. Pregnancy stages were estimated relative to published developmental milestones (Hickford et al. 2009: Tyndale-Biscoe and Renfree 1987). Swamp wallaby pouch young were removed from our research colony animals and killed by sodium pentobarbitone injection $(60 \mathrm{mg} / \mathrm{ml} ; 150 \mathrm{mg} / \mathrm{kg})$ under approval from the University of Melbourne Institutional Animal Ethics Committees. All experiments conformed to the Australian National Health and Medical Research Council (2013) guidelines.

\section{Tissue collection}

Tissues, including gravid and non-gravid endometrium (stages: diapause, and days 5, 11, 17, 21 and 26 of reactivated pregnancy), bilaminar and trilaminar placenta (stages: days 18, 19, 21, 24 and 25 of reactivated pregnancy), and late stage fetus (day 20 of pregnancy), were dissected from pregnant animals. Adult thymus tissue was used as a positive control. Tissues were placed in RNase-free cryovials and snap-frozen in liquid nitrogen immediately post-mortem. Samples were stored permanently at $-80^{\circ} \mathrm{C}$ until RNA extraction.

\section{RNA extraction and cDNA synthesis}

Total RNA was extracted from tri- and bi-laminar placenta, gravid and non-gravid endometrium, whole fetus and thymus using TriReagent (Ambion, USA) according to the manufacturer's protocol. Total RNA was then DNase-treated with DNAfree (Ambion, USA) according to the manufacturer's instructions and stored at $-80^{\circ} \mathrm{C}$ until further required. Oligo(dT)-primed cDNA was then synthesized from total RNA samples using the Superscript III First-Strand-Synthesis system for RT-PCR (Invitrogen, USA) using the manufacturer's protocol.

\section{PCR, DNA cloning and sequence analysis}

Polymerase chain reactions (PCRs) contained $12.5 \mu \mathrm{L}$ GoTaq Green DNA master mix (Promega, USA), $1 \mu \mathrm{L}$ of undiluted cDNA, $1 \mu \mathrm{L}$ of $10 \mu \mathrm{M}$ forward and reverse primer, respectively, and 9.5 $\mu \mathrm{L}$ of nuclease-free $\mathrm{H}_{2} \mathrm{O}(25 \mu \mathrm{L}$ total volume). Both $\mathrm{MHC}$ unlinked and linked primer combinations, putative classical and non-classical genes respectively, were from Siddle et al [36]. They include MHC-I unlinked (Fwd: CACTCCATGAGGTATTTCGACA, Rev: GGCTCAGGCAG-CCCCTCGTGC), MHC linked class I Maeu-UE (Fwd: ATGTGCCTGCAGAAAGTGTCTGCG， Rev： ATGGTTCAGGGCTC-CTGAGTTCC), MHC 
linked class I Маеи-UK (Fwd: AGTA-GTTAGAGAGACGGAGCACAC, Rev: GTACTTCTGCAGCCATTCAGT), MHC linked Maeu-UO (Fwd: CAAGAGATACCAGATtACTGGGA, Rev: CGTTCCCAGCGATCCAACTTAGA), and MHC linked $\quad$ Maeu-UM (Fwd: GCGGGCCCAGACTGGGGTTAGAG, Rev: ACGTTTAGGGCCACGTTGTCCA-AT). PCR conditions were identical to those described by Siddle et al (Siddle et al. 2009) apart from the initial denaturation, which was held at $95^{\circ} \mathrm{C}$ for 5 minutes and the final extension of $72^{\circ} \mathrm{C}$ for 5 minutes. B2-microglobulin primers (F: Fwd: ATGGCTCGCAACTTCTTCCT; Rv: TTAGTTGTTTTCTGCATCCC) were substituted in a similar PCR reaction and incubated in a thermocycler under the following conditions, 95oC for 5 minutes, then five cycles of $95 \mathrm{oC}$ for $30 \mathrm{~s}, 50-45 \mathrm{oC}$ for $30 \mathrm{~s}$ and $72 \mathrm{oC}$ for 1 minute. A further 30 cycles of $95 \mathrm{oC}$ for $30 \mathrm{~s}, 45 \mathrm{oC}$ for $30 \mathrm{~s}$ and $72 \mathrm{oC}$ for 1 minute was then performed. Swamp wallaby Wabi-UM was amplified using conserved primers designed from an alignment of opossum, Tasmanian devil and tammar wallaby $U M$ sequences (Fwd: GCTACGACGGACAAGACAAACTC, Rev: ACT-GTGGCTCCCATTTCAAGG; accession number: KM269669). PCR amplification conditions for Wabi-UM consisted of $95^{\circ} \mathrm{C}$ for 5 minutes, 30 cycles of $95^{\circ} \mathrm{C}$ for 30 seconds, $60^{\circ} \mathrm{C}$ for 30 seconds, and $72^{\circ} \mathrm{C}$ for 1 minute. A final extension step of $72^{\circ} \mathrm{C}$ for 5 minutes completed the reaction.

PCR products were ligated into the $p G E M-T$ easy vector system (Promega, USA) and transformed into JM109 competent cells (Promega, USA) using the manufacturer's protocols. Positive clones were identified using IPTG/XGAL selection media (Fermentas, Germany) and multiple clones were isolated and sequenced in forward and reverse direction using SP6 and T7 primers. Individual sequence chromatographs were manually checked for sequence accuracy.

\section{Sequence analysis of placental unlinked MHC-I transcripts}

MHC-I unlinked primers universally amplify a range of MHC-I unlinked genes. To understand which sequences were expressed in the placenta and at what ratio, 10 positive clones from individual PCR amplifications in bilaminar (BOM) and trilaminar (TOM) yolk sac placenta were sequenced at days 18, 19, 21, 24, and 25 of the 26-day pregnancy. Each pair of BOM and TOM at each stage of pregnancy represents one individual. We also sequenced 10 clones from the liver cDNA of one individual as a comparison ( 6 individuals and 110 clones in total). Clones not present in both BOM and TOM were removed from the analysis (19/110) to avoid possible chimeras or PCR induced errors. MHC-I unlinked transcripts were aligned using Clustal-W in Mega 5.05 (Tamura et al. 2011). These sequences have been submitted to Genbank with the accession numbers (KM269559-KM269668). 


\section{Phylogenetic analyses}

The evolutionary relationship between MHC-I unlinked genes Maeu-UA (CU457750), -UB (CU302514), -UC (CU302420), -UD (CU302406), -UF (CU302408), -UH (CU302408), -UI (CU302372), -UJ (CU302374), and -UN (CU302373) and expressed MHC-I unlinked transcripts ( $\alpha 1 / \alpha 2$ domains, 500 nucleotides) were compared using the neighbour-joining, maximum likelihood, maximum parsimony and minimum evolution methods in Mega 5.05 (Tamura et al. 2011). Maeu-UP (CU463996), a phylogenetically ancestral MHC-I linked gene, was also included in the analysis (Siddle et al. 2009). Previously published tammar wallaby MHC-I transcripts were included in the analysis, including Maeu-1*01 (DQ304109), Maeu-1*02 (DQ304110), Maeu-I*03 (DQ304111) and Maeu-I*07-17 (FJ238079-FJ238088), in order to further reconcile gene/transcript relationships (Siddle et al. 2006; 2009). The percentage of replicate trees in which the associated sequences clustered together in the bootstrap test (1000 replicates) is shown next to branches (Felsenstein 1985). Phylogenetic trees display the bootstrap consensus topology. The evolutionary distances were computed using the Jukes-Cantor method (Jukes and Cantor 1969), which was used previously to estimate evolutionary rates for MHC-I $\alpha 1 / 2$ domains (Siddle et al. 2009). All positions containing gaps or missing data were eliminated.

To determine orthologous relationships between marsupial MHC-I linked genes, 487 bp of the $\alpha 2$ and $\alpha 3$ domains of the $-U M$ gene were compared using the same analysis described above with the Maeu-UA gene included as an outgroup. Published sequences used in the analysis included: tammar wallaby Maeu-UE (CU463962), Maeu-UK (CU463018), Maeu-UM (CU463962) and Maeu-UO (CU466525); Tasmanian devil (Sarcophilus harrisii) Saha-UK (KJ713550) and Saha-UM (KJ713551); and grey short-tailed opossum (Monodelphis domestica) Modo-UE, Modo-UK, ModoUM and Modo-UO (http://bioinf.wehi.edu.au/opossum/seq/Class_I.fa).

\section{Results and Discussion}

\section{Expression of MHC-I unlinked genes in tammar fetal and reproductive tissues}

Tammar MHC-I unlinked genes were expressed in both TOM and BOM of the placenta from day 18 to day 25 of pregnancy and in the fetus at day 20 of pregnancy (Figure 1). Expression was also observed in gravid and non-gravid endometrium during diapause and early pregnancy (day 5), and in gravid endometrium at days 11, 17, 21 and 26 of pregnancy (Figure 1). MHC-I mRNA expression appeared reduced relative to GAPDH mRNA in BOM at days 24 and 25 of pregnancy, TOM at day 25 of pregnancy and endometrium at days 17 and 21 of pregnancy, which may indicate 
fine-scale regulation in these tissues during late gestation. However, further studies using quantitative PCR for specific MHC-I genes over pregnancy will be required to clarify this. Both endometrium and placenta samples also expressed $\beta 2$-microglobulin, an important molecule that is non-covalently linked to the MHC-I heavy chain, stabilising the trimer.

MHC-I unlinked transcripts cloned from placenta were phylogenetically most similar to the class I genes Maeu-UC (50/100 clones), Maeu-UD (34 clones), and Maeu-UA (7/100 clones) (Figure 2). The high level of polymorphism within the PBR ( $\alpha 1 / 2$ domain) of some MHC-I transcripts resulted in very low bootstrap support for specific gene/transcript groupings. For example, there was very low bootstrap support (<10\%) dividing Maeu-UA and Maeu-UC genes and associated transcripts. Alternative phylogenetic models including maximum likelihood, maximum parsimony and minimum evolution did not improve the bootstrap support observed using the neighbour-joining phylogeny (data not shown). Maximum parsimony and likelihood methods also changed the consensus tree topology so that the Maeu-UA and Maeu-UD genes and associated transcripts were positioned on common sub-branches. However, these alternate topologies did not show strong bootstrap support $(<20 \%)$ relative to the neighbour-joining and minimum evolution models $(50 \%$; Figure 2).

Two unique transcripts grouping phylogenetically with Maeu-UC were identified from three different individuals (d18, d24 and d25 BOM and TOM) suggesting that both alleles of the Maeu$U C$ locus may be expressed at the fetal-maternal interface in the tammar. The higher total number of Maeu-UC transcripts isolated relative to the other putative classical class I genes (Maeu-UA/UB) may indicate an expression bias in the placenta similar to $H L A-C$ in human trophoblast. However, this could also be due to the non-specificity of the MHC-unlinked primer sequence and the possibility that they preferentially amplify Maeu-UC in this population of tammars. Further studies using specific primers for Maeu-UA/UD will clarify this.

Transcripts similar to the Maeu-UD gene represented a much higher proportion of transcripts, and by extension a higher level of expression, than observed previously in blood and thymus [36]. For one tissue sample (day 21 placenta), Maeu-UD transcripts included $100 \%$ and $70 \%$ of total BOM and TOM MHC-I sequences, respectively (Table I). Placental Maeu-UD differed by only one amino acid substitution between the three individuals that showed expression (threonine to asparagine at position 81 of the $\alpha 1 / 2$ domain) suggesting it is a non-classical gene. The Maeu-UD gene described previously also differed at this site (threonine to asparagine) and one other (methionine to leucine at position 3). Interestingly, the Maeu-I*14 (FJ238084) transcript, published previously and thought to 
be the product of Maeu-UD [36], was very different from both the Maeu-UD gene and the placental transcripts, encoding 22 and 31 amino acid differences, respectively (Online Resource 1). Thus, МаeuI*14 may be the product of an alternative gene given the nucleotide conservation and monomorphic nature of placental-UD from three other individuals.

There were no transcripts isolated similar to Maeu-UB, -UF, -UI, -UJ, -UH or -UN. However, after closer inspection of the full-length sequence of these genes we found that the MHC-I unlinked universal primer pair would not have amplified transcripts from these loci, and therefore they must have been isolated previously using 5' RACE PCR in conjunction with the reverse primer (Siddle et al. 2009). Thus, these other MHC-I unlinked genes may still be expressed by placenta but not detected in our PCRs. This may also explain why Maeu-UD transcripts were not amplified in some individuals, as the Maeu-UD gene contains at least one nucleotide mis-match at the annealing site of the universal forward primer.

\section{Expression of MHC-I linked genes in tammar fetal and reproductive tissues}

Putative non-classical MHC-I genes (MHC-linked in the wallaby) displayed tissue-specifc expression in the embryo and reproductive tract. Maeu-UK, a ubiquitously expressed monomorphic MHC-I sequence, was present at high levels in all tissues tested (Figure 3). Maeu-UE and -UO, which were previously found to be restricted to the thymus, were expressed in the whole fetus at day 20 of pregnancy (Figure 3). However, this may indicate expression from thymic progenitor cells. Interestingly, Maeu-UM, a highly conserved marsupial-specific non-classical gene, was highly expressed in the embryo and placenta but not by the apposing gravid endometrium (Figure 3). This gene was previously detected in adult thymus and at very low levels in lung and spleen (Siddle et al. 2009). Sequence conservation and high expression in the embryo and fetal membranes suggests that Maeu-UM might have a specific role in marsupial pregnancy.

The opossum expresses three isoforms of -UM (full length, exon 3 deleted and exons 5, 6 and 7 deleted transcripts). Modo-UM contains an unpaired cysteine in the $\alpha 1$ domain, which is unusual for MHC-I molecules but consistent with homodimer formation by non-classical MHC-I, including human HLA-G (Baker et al. 2008). Importantly, the position and presence of this unpaired cysteine is conserved in all marsupial -UM sequences (wallaby, devil and opossum; Online Resource 2). Thus, an immunomodulatory role for non-classical MHC, possibly to inhibit natural killer cells, in the mammalian placenta may predate the divergence of therian mammals. However, this requires further investigation. 
The putative non-classical MHC-I gene, -UM, is conserved in marsupials

We developed conserved primers to amplify 489 bp of the orthologous - UM mRNA from a fourth marsupial, the swamp wallaby (Wallabia bicolor; Wabi-UM). Wabi-UM was $99 \%$ identical to Maeu-UM and contained five nucleotide substitutions of which three were non-synonymous and predicted amino acid substitutions at positions 39 (methionine to isoleucine), 50 (isoleucine to valine) and 76 (arginine to histidine) with $M a e u-U M$, respectively. The macropodid $U M$ s (tammar and swamp wallaby) shared approximately $83 \%$ nucleotide identity and $80 \%$ amino acid identity with opossum and Tasmanian devil UMs.

Phylogenetic analysis of marsupial -UM sequences showed strong bootstrap support for a common orthology (bootstrap value: 100) relative to other putative non-classical MHC genes (-UE, $-U O$ and -UK; Figure 4). Sequence conservation of a non-classical MHC gene over such distant marsupial lineages suggests that the $-U M$ gene may have gained a specific immunological function. While the $-U K$ gene is similarly conserved between marsupial lineages, it is expressed ubiquitously in all tissues suggesting it may have a more generalised function.

Given that some eutherian species such as rodents express classical MHC-I genes in trophoblast, yet others such as humans do not, the presence of both classical and non-classical genes in trophoblast of the tammar wallaby, a distant ancestor, suggests that the expression of both types is the ancestral state in mammals, while humans have lost placental expression secondarily. Thus, expression of self-molecules is likely an important developmental event during embryonic/fetal life in developing mammals. Interestingly, the unlinked gene Maeu-UD appears to be a highly expressed, monomorphic, placenta-specific MHC-I gene. Future work will determine how the expression of this gene changes through pregnancy and to which cells expression is localised.

\section{Conclusions}

We have demonstrated that putative classical MHC-I genes are expressed for the whole period of uterine placental apposition, and at similar level to those of non-placental tissues including liver, blood and thymus. The presence of a putative non-classical, placenta-specific MHC-I gene Maeu$U D$ suggests there is fundamental role for monomorphic MHC-I in modulating the immune system at the fetal-maternal interface in mammals. The high conservation of mRNA expression of classical and non-classical MHC-I genes between extant marsupial and eutherian mammals supports their importance in the evolution of the ancestral therian placenta and survival of the mammalian fetus at the maternal-fetal interface. 


\section{Authors' contributions}

$\mathrm{BD}, \mathrm{TBH}, \mathrm{MBR}$ and BRM designed the project. BRM and MBR collected and extracted the RNA from tammar wallaby placental tissues. IB isolated the sequences from tammar placenta and performed and checked PCR transcripts. SRF recapitulated all class I genes from published BAC libraries using bioinformatic resources and assisted with phylogenetic analysis and universal $-U M$ primer design. All authors edited and approved the final manuscript.

\section{Acknowledgements}

Many thanks to Mrs Jette Ziep and Ms Tanja Noventa for assistance in the laboratory, and to Prof Joerns Fickel for technical assistance with DNA sequencing. This project was funded by a PAKT grant from the Leibniz Gemeinschaft to BD, a Humboldt Postdoctoral Fellowship to BRM, an Australian Research Council Discovery Outstanding Research Award to MBR. Thank you to the Dahlem Research School for supporting this research with a travel grant to Mrs Ina Buentjen.

\section{Conflict of interest}

The authors declare that they have no conflict of interest.

\section{List of abbreviations}

MHC-I: Major Histocompatibility Complex Class I

PBR: protein binding region

a1/2: alpha $1 \& 2$ domain

BOM: bilaminar omphalopleure

TOM: trilaminar omphalopleure

cDNA: complimentary DNA 


\section{References}

Amoroso EC (1959) Comparative anatomy of the placenta. Ann N Y Acad Sci 73:855-872.

Andrews DM, Sullivan LC, Baschuk N, Chan CJ, Berry R, Cotterell CL, Lin J, Halse H, Watt SV, Pousine-Larent J, Wang CR, Scalzo AA, Yokoyama WM, Rossjohn J, Brooks AG, Smyth MJ (2012) Recognition of the non-classical class I molecule H2-M3 by the receptor Ly49A regulates the licensing and activation of NK cells. Nature Immunol 13:1171-1177.

Bainbridge DRJ (2000) Evolution of mammalian pregnancy in the presence of the maternal immune system. Rev Reprod 5:67-74.

Baker ML, Melman D, Huntley J, Miller RD (2008) Evolution of the opossum major histocompatibility complex: evidence for diverse alternative splice patterns and low polymorphism among class I genes. Immunol 128:e418-e431.

Belov K, Deakin JE, Papenfuss AT, Baker ML, Melman SD, Siddle HV, Gouin N, Goode DL, Sargeant TJ, Robinson MD, Wakefield MJ, Mahony S, Cross JG, Benos PV, Samollow PB, Speed TP, Graves JA, Miller RD (2006) Reconstructing an ancestral mammalian immune supercomplex from a marsupial major histocompatibility complex. PLoS Biol 4:e46.

Birch J, Murphy L, MacHugh ND, Ellis SA (2006) Generation and maintenance in the cattle MHC class I region. Immunogenetics 58:670-679.

Bjorkman PJ, Parham P (1990) Structure, function, and diversity of class I major histocompatibility complex molecules. Annu Rev Biochem 59:253-288.

Blaschitz A, Hutter H, Dohr G (2001) HLA Class I protein expression in the human placenta. Early Pregnancy 5:67-69.

Cao W, Brenner CA, Alikani M, Cohen J, Warner CM (1999) Search for human homologue of the mouse Ped gene. Mol Human Reprod 5:541-547.

Davies CJ, Fisher PJ, Schlafer DH (1990) Temporal and regional regulation of major histocompatibility complex class I expression at the bovine uterine/placental interface. Placenta 21:194-202.

Donaldson WL, Zhang CH, Oriol JG, Antczak DF (1990) Invasive equine trophoblast expresses class I Major Histocompatibility Complex antigens. Development 110:63-70.

Felsenstein J (1985) Confidence limits on phylogenies: An approach using the bootstrap. Evolution 39:783-791.

Freyer C, Zeller U, Renfree MB (2002) Ultrastructure of the placenta of the tammar wallaby, Macropus eugenii: comparison with the grey short-tailed opossum, Monodelphis domestica. J Anat 201:101-119.

Gaycken U, Shabahang M, Meyer JN, Glodek P (1994) Molecular characterization of the porcine MHC class I region. Anim Genet 25:537-539. 
Günther E, Walter L (2001) The major histocompatibility complex of the rat (Rattus norvegicus). Immunogenetics 53:520-542.

Gustafson AL, Tallmadge RL, Ramlachlan N, Miller D, Bird H, Antczak DF, Raudsepp T, Chowdhary BP, Skow LC (2003) An ordered BAC contig map of the equine major histocompatibility complex. Cytogenet Genome Res 102:1-4.

Hedrick PW, Thomson G (1988) Maternal-fetal interactions and the maintenance of HLA polymorphism. Genetics 104:449-456.

Hickford D, Frankenberg S, Renfree MB (2009) The tammar wallaby, Macropus eugenii: A model kangaroo for the study of developmental and reproductive biology. Cold Spring Harb Protoc 12:449-494.

Hughes A, Nei M (1988) Pattern of nucleotide substitution at the major histocompatibility complex class I loci reveals overdominant selection. Nature 335:167-170.

Hulpke S, Tampé R (2013) The MHC I loading complex: a multitasking machinery in adaptive immunity. Trends Biochem Sci 38:412-420.

Jukes TH, Cantor CR (1969) Evolution of protein molecules. In: Munro HN (ed) Mammalian Protein Metabolism. Academic Press, New York, pp 21-132.

Luo ZX, Yuan CX, Meng QJ, Ji Q (2011) A Jurassic eutherian mammal and divergence of marsupials and placentals. Nature 476:442-445.

Madeja Z, Yadi H, Apps R, Boulenouar S, Roper SJ, Gardner L, Moffett A, Colucci F, Hemberger M (2011) Paternal MHC expression on mouse trophoblast affects uterine vascularization and fetal growth. Proc Natl Acad Sci USA 108:4012-4017.

Medawar PB (1953) Biological problems of skin surgery. J Int Chir 13:385-391.

Nei M, Gu X, Sitnikova T (1997) Evolution by the birth-and-death process in multigene families of the vertebrate immune system. Proc Natl Acad Sci USA 94:7799-7806.

Nonaka M, Namikawa C, Kato Y, Sasaki M, Salter-Cid L, Flajnick MF (1997) Major histocompatibility complex gene mapping in the amphibian Xenopus implies a primordial organization. Proc Natl Acad Sci USA 94:5789-5791.

Otting N, Heijmans CM, Noort RC, de Groot NG, Doxiadis GG, van Rood JJ, Watkins DI, Bontrop RE (2005) Unparalleled complexity of the MHC class I region in rhesus macaques. Proc Natl Acad Sci USA 102:1626-1631.

Parham P, Norman PJ, Abi-Rached L, Hilton HG, Guethlein LA (2012) Review: Immunogenetics of human placentation. Placenta 33 Suppl:S71-80.

Pyo CW, Williams LM, Moore Y, Hyodo H, Li SS, Zhao LP, Sageshima N, Ishitani A, Geraghty DE (2006) HLA-E, HLA-F, and HLA-G polymorphism: genomic sequence defines haplotype structure and variation spanning the non-classical class I genes. Immunogenetics 58:241-251. 
Redman CW, McMichael AJ, Stirrat GM, Sunderland CA, Ting A (1984) Class 1 major histocompatibility complex antigens on human extra-villous trophoblast. Immunology 52:457488.

Renfree MB (1972) Influence of the embryo on the marsupial uterus. Nature 240:475-477.

Renfree MB, Papenfuss AT, Shaw G, Pask AJ (2009) Eggs, embryos and the evolution of imprinting: insights from the platypus genome. Reprod Fertil Dev 21:935-942.

Rogers JH (1985) Mouse histocompatibility-related genes are not conserved in other mammals. Embo J 4:749-753.

Sambrook JG, Figueroa F, Beck S (2005) A genome wide survey of Major Histocompatibility Complex (MHC) genes and their paralogues in zebrafish. BMC Genomics 6:152.

Selwood L (2000) Marsupial egg and embryo coats. Cells Tissues Organs 166:208-219.

Shiina T, Shimizu S, Hosomichi K, Kohara S, Watanabe S, Hanzawa K, Beck S, Kulski JK, Inoko H (2004) Comparative genomic analysis of two avain (quail and chicken) MHC regions. J Immunol 172:6751-6763.

Siddle HV, Deakin JE, Baker ML, Miller RD, Belov K (2006) Isolation of major histocompatibility complex Class I genes from the tammar wallaby (Macropus eugenii). Immunogenetics 58:487493.

Siddle HV, Deakin JE, Coggill P, Hart E, Cheng Y, Wong ES, Harrow J, Beck S, Belov K (2009) MHC-linked and un-linked class I genes in the wallaby. BMC Genomics 10:310.

Siddle HV, Marzec J, Cheng Y, Jones M, Belov K (2010) MHC gene copy number variation in Tasmanian devils: implications for the spread of a contagious cancer. Proc Biol Sci 277: 20012006.

Tallmadge RL, Lear TL, Antczak DF (2005) Genomic characterization of MHC class I genes of the horse. Immunogenetics 57:763-774.

Tamura K, Peterson D, Peterson N, Stecher G, Nei M, Kumar S (2011) MEGA5: Molecular Evolutionary Genetics Analysis using Maximum Likelihood, Evolutionary Distance and Maximum Parsimony Methods. Mol Biol Evol 28:2731-2739.

Tyndale-Biscoe CH, Renfree MB (1987) Reproductive Physiology of Marsupials. Cambridge University Press, Cambridge.

Warner CM, Gollnick SO, Flaherty L, Goldbard SB (1987) Analysis of Qa-2 antigen expression by preimplantation mouse embryos: Possible relationship to the Ped gene product. Biol Reprod 36:611-616.

Yuhki N, Beck T, Stephens R, Neelam B, O’Brien SJ (2007) Comparative structure of human, dog, and cat MHC: HLA, DLA, and FLA. J Hered 98:390-399. 
Zinkernagel RM, Doherty PC (1974) Immunological surveillance against altered self components by sensitised T lymphocytes in lymphocytic choriomeningitis. Nature 251:547-548. 


\section{Figure captions}

\section{Figure 1: Unlinked MHC-I and ß2-microglobulin expression in tammar reproductive tissues}

RT-PCR using primers specific to unlinked, putative classical, MHC-I shows strong expression in endometrium during diapause, gravid and non-gravid stages (A and B) and in both bilaminar $(\mathrm{BOM})$ and trilaminar (TOM) yolk sac placenta (C) at all stages after shell coat rupture (day 18-25 of pregnancy). $\beta 2$-microglobulin, a key molecule that is non-covalently associated with the MHC-I heavy chain stabilising the trimer, was also present in BOM, TOM and endometrium throughout the attachment phase of pregnancy (D). Gr: gravid; n-gr: non-gravid; endo: endometrium; BOM: bilaminar placenta; TOM: trilaminar placenta; Di: diapause stage; $\beta 2 \mathrm{~m}$ : $\beta 2$-microglobulin).

Figure 2: Phylogenetic relationship between tammar wallaby placental expressed MHC-I unlinked transcripts and MHC-I unlinked genes and published transcripts

The neighbour-joining tree indicates the majority of unlinked MHC-I transcripts expressed in tammar placenta are similar to the genes Маeи-UA, Маеи-UC and Maeu-UD. However, basal divisions between $-U A$ and $-U C$ like genes and transcripts were supported by a low bootstrap value (8). A high proportion of transcripts grouped with the Maeu-UD gene (34/100 clones) relative to previous studies, and appeared monomorphic within the PBR between all individuals in which expression was detected. Transcript colours denote specific individuals in the legend. The bracketed number indicates the number of identical clones. Bracketed sequences indicate the average percentage nucleotide identity among the group.

\section{Figure 3: Linked MHC-I expression in tammar reproductive tissues.}

Linked, non-classical, MHC-I genes showed tissue-specific expression in the tammar placenta by RT-PCR. Маeu-UK was expressed in all tissues tested, similar to its ubiquitous tissue profile observed previously [36]. Maeu-UE and -UO were expressed in the fetus, which may originate from thymic progenitor cells, but not in the endometrium or placenta. Interestingly, Маeu-UM was expressed strongly in the bilaminar and trilaminar yolk sac placenta and in the fetus, , but not in the endometrium, which may indicate a pregnancy-specific role for this molecule.

Figure 4: Phylogenetic relationship between putative non-classical MHC-I genes in different marsupials

Phylogenetic analysis [method] provides strong bootstrap support (100) for an orthologous relationship between marsupial - $U M$ genes. The UK non-classical gene also shows this relationship between marsupial lineages. Tammar $-U E$ appears to have arisen from a duplicated ancestral $-U O$ 
gene after the divergence of Macropodids from other lineages, since - $U E$ was not identified in opossum or devil.

\section{Table captions}

Table I: Proportion of MHC-I unlinked transcripts present in tammar placental tissue.

Unlinked class I transcripts in tammar placenta were most similar to five class I genes (Maeu-UA, Maeu-UC, and Мaeu-UD). Interestingly, Maeu-UD like genes represented 34/100 sequenced placental transcripts. This may indicate that Maeu-UD plays an imporant role in the tammar placenta. There were no specific trends in transcripts observed for tissue type (BOM/TOM) or stage of pregnancy (d18-25).

\section{Online Resources}

\section{Online Resource 1: Amino acid sequence comparison of tammar Maeu-UD like transcripts}

Maeu-UD transcripts were present in placental tissue at days 18, 21 and 25 of pregnancy and were 99.87\% identical to the Maeu-UD gene (two nucleotide differences: yellow boxes). A single liver transcript also grouped with the Maeu-UD gene phylogenetically, but this sequence contained numerous nucleotide differences that encode 22 amino acid substitutions relative to Maeu-UD. The lack of variability in placental Maeu-UD transcripts from three different individuals suggests that the liver transcript is the product of an alternative gene.

\section{Online Resource 2: Amino acid sequence comparison of marsupial MHC-I -UM genes.}

The MHC-I -UM gene is highly conserved in marsupials, sharing approximately $80 \%$ amino acid identity between opossum, devil and wallaby. The $\alpha 1$ and $\alpha 2$ domains (underlined in red and blue, respectively) are particularly highly conserved and contain the protein-binding region that is normally highly polymorphic in classical MHC-I genes. The cysteine residue in the $\alpha 1$ domain (yellow box), identified as an unpaired cysteine in opossum, is also conserved in all marsupials. 
Table I: Numbers of MHC-I unlinked transcripts present in tammar placental tissue.

\begin{tabular}{cccccc}
\hline Animal & Tissue & Maeu-UC like & Maeu-UA like & Maeu-UD-like & Not included \\
\hline 1. Day 18 preg & BOM/TOM & 8 & 0 & 8 & 4 \\
2. Day 19 preg & BOM/TOM & 15 & 0 & 0 & 5 \\
3. Day 21 preg & BOM/TOM & 0 & 0 & 17 & 3 \\
4. Day 24 preg & BOM/TOM & 13 & 7 & 0 & 3 \\
5. Day 25 preg & BOM/TOM & 8 & 0 & 9 & 4 \\
6. Day 25 preg & Liver & 6 & 0 & 0 & 19 \\
\hline Total & & 50 & 7 & 34 & \\
\hline
\end{tabular}




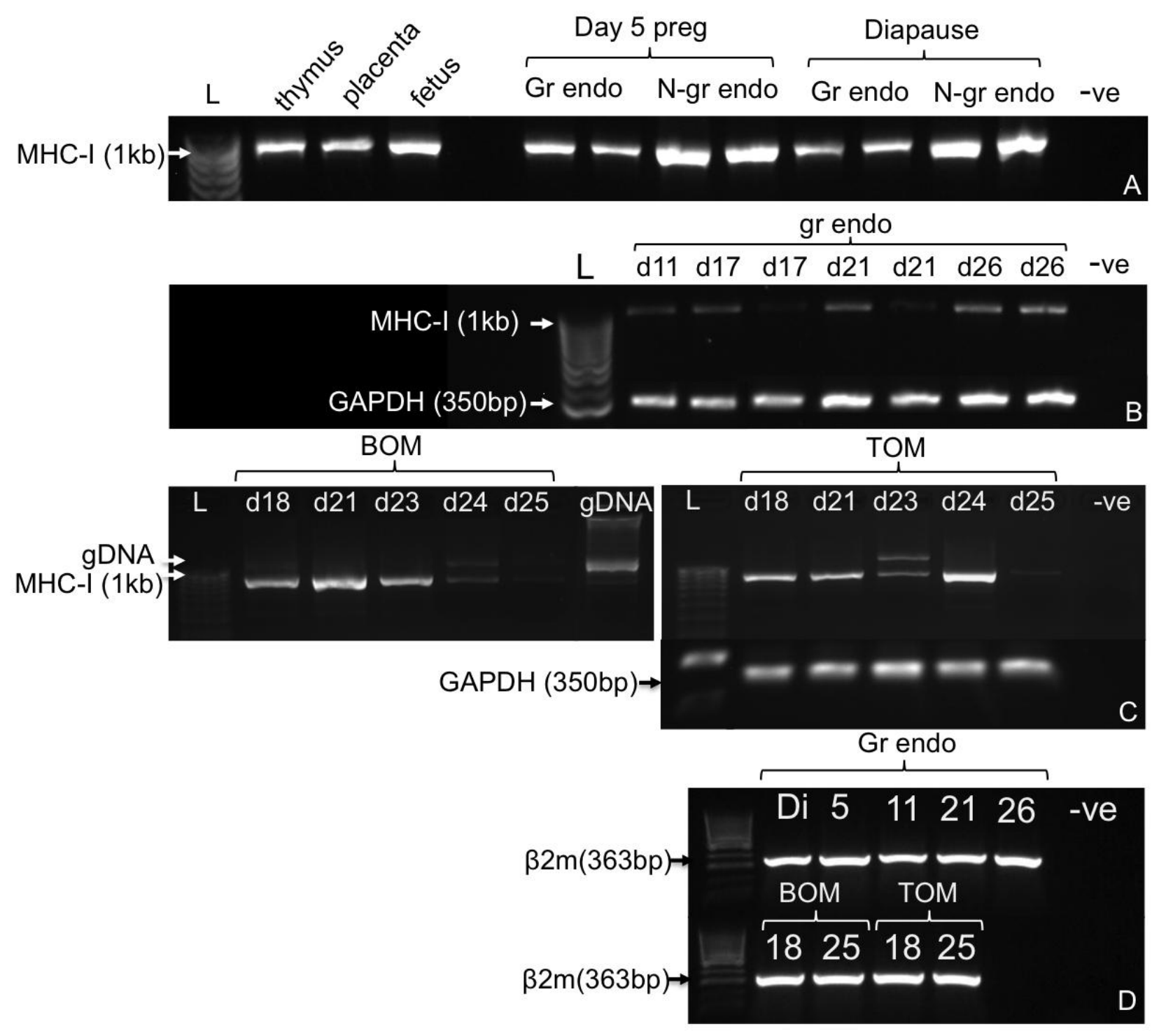




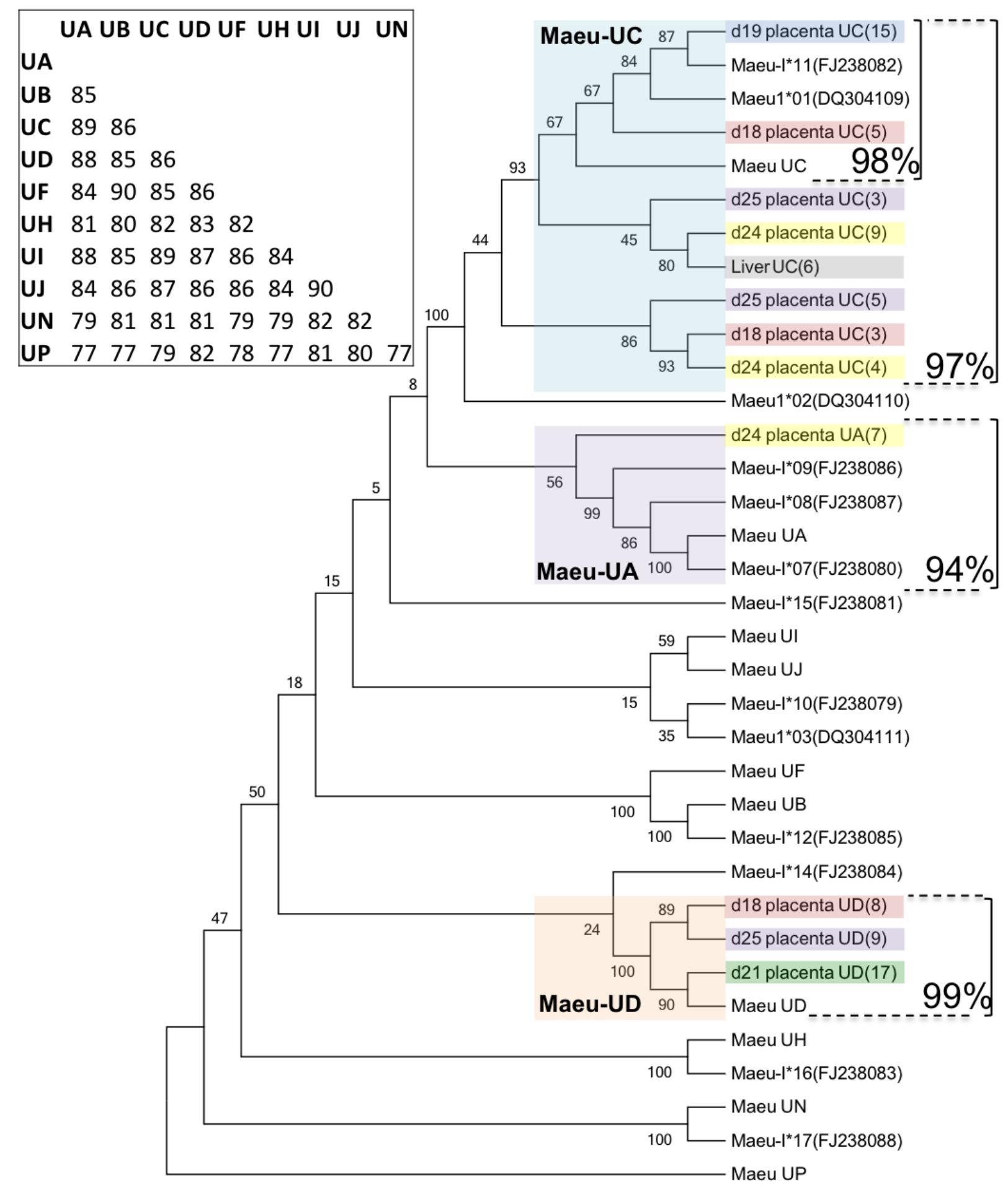




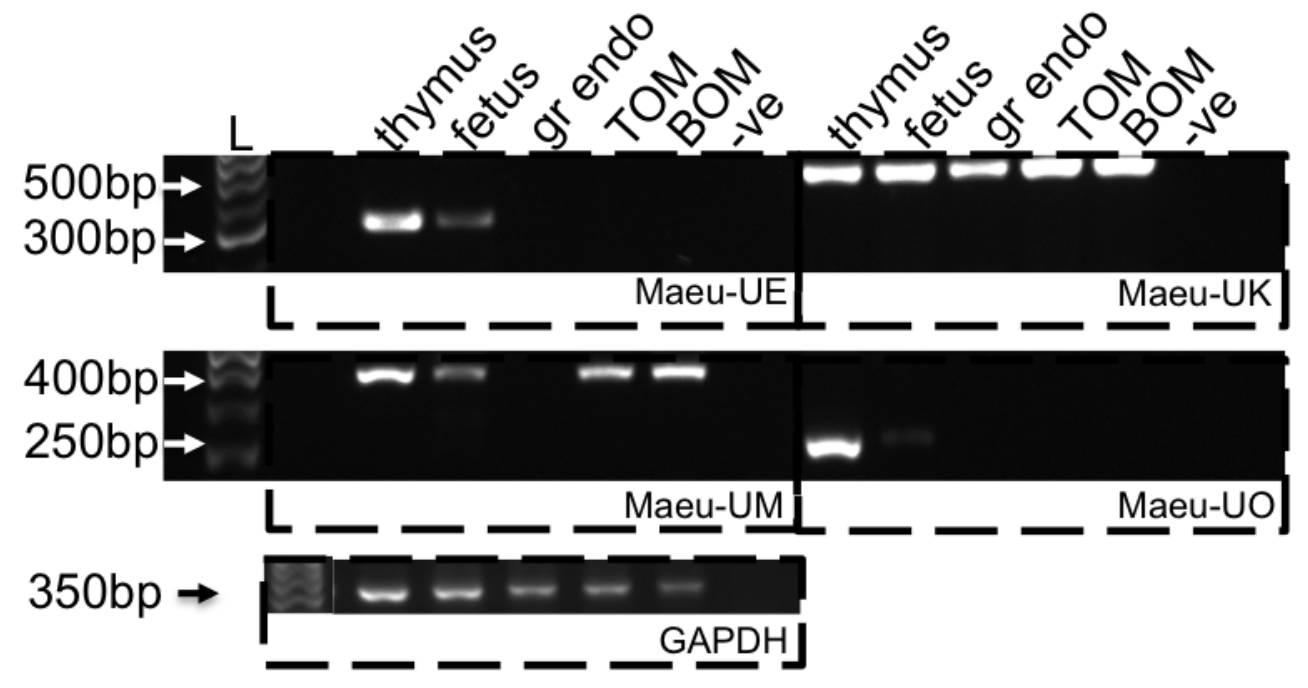




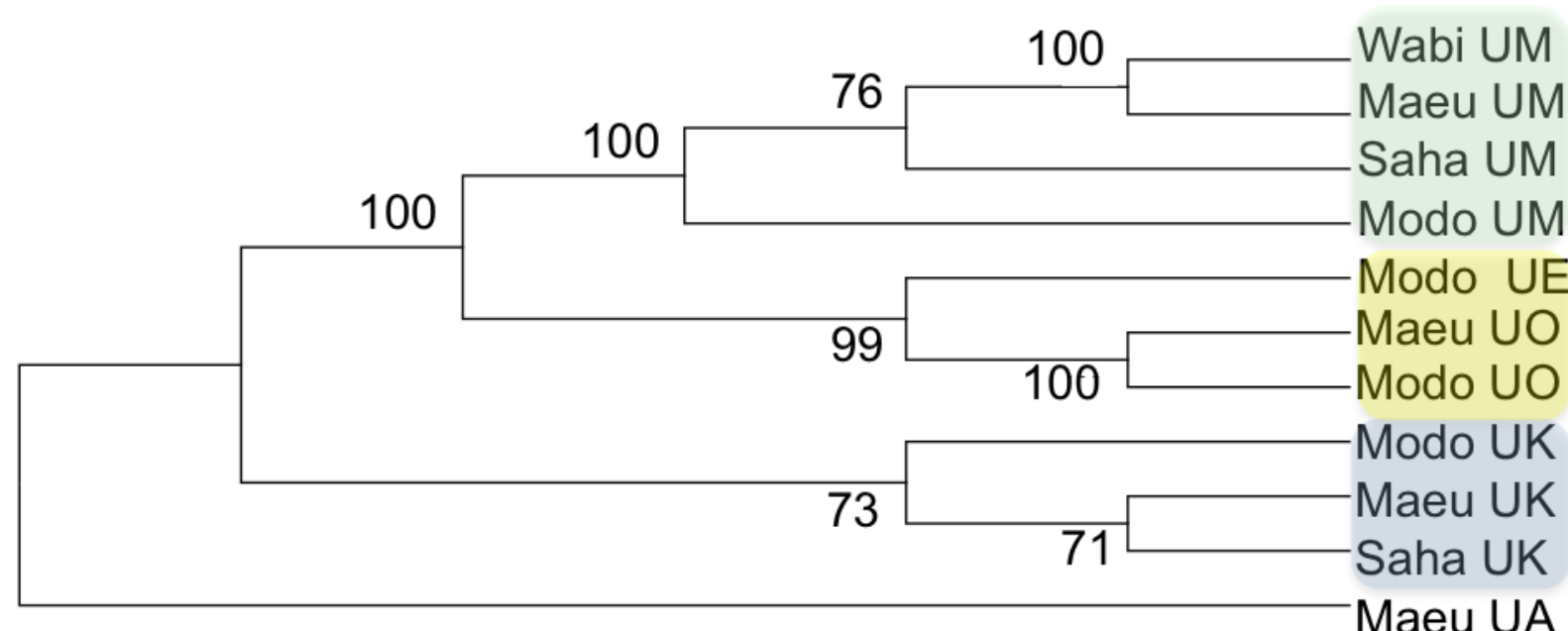




\section{University Library}

\section{- M M I N E R VA \\ A gateway to Melbourne's research publications}

Minerva Access is the Institutional Repository of The University of Melbourne

Author/s:

Buentjen, I;Drews, B;Frankenberg, SR;Hildebrandt, TB;Renfree, MB;Menzies, BR

Title:

Characterisation of major histocompatibility complex class I genes at the fetal-maternal interface of marsupials

Date:

2015-07-01

Citation:

Buentjen, I., Drews, B., Frankenberg, S. R., Hildebrandt, T. B., Renfree, M. B. \& Menzies, B. R. (2015). Characterisation of major histocompatibility complex class I genes at the fetal-maternal interface of marsupials. IMMUNOGENETICS, 67 (7), pp.385-393. https:// doi.org/10.1007/s00251-015-0842-5.

Persistent Link:

http://hdl.handle.net/11343/283223 